\title{
Pankreaszysten bei Kindern und Jugendlichen
}

\author{
Winfried Barthlen und Dietrich von Schweinitz
}

\section{Inhalt}

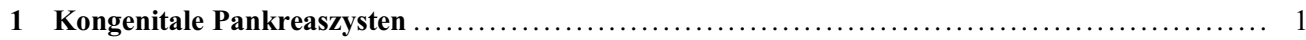

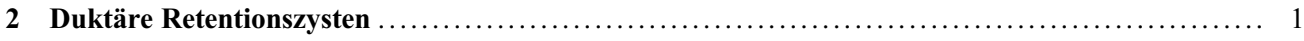

Literatur

Da Pankreaszysten bildmorphologisch oft nur schwierig von zystischen Tumoren differenziert werden können, sollten sie reseziert werden.

\section{$1 \quad$ Kongenitale Pankreaszysten}

Die sehr seltenen kongenitalen dysontogenetischen Pankreaszysten sind differenzialdiagnostisch von anderen zystischen Veränderungen des Pankreas abzugrenzen, besonders von den zystischen Tumoren (Kap. „Kongenitale Anomalien des Pankreas“, Kap. „Pankreatitis bei Kindern und Jugendlichen“, Kap. \,Tumoren des Pankreas bei Kindern und Jugendlichen"). Meistens werden sie bereits im frühen Kindesalter als Zufallsbefund bei einer Bildgebung aus anderer Ursache entdeckt. Die Zysten verursachen meist keine Symptome und beeinträchtigen das umgebende Pankreasgewebe nicht. Der Inhalt enthält keine Pankreasenzyme und es besteht keine Verbindung zum Gangsystem. Über Spontanverläufe von kongenitalen Pankreaszysten über längere Zeit liegen keine Daten vor.

W. Barthlen $(\bowtie)$

Klinik und Poliklinik für Kinderchirurgie, Universitätsmedizin

Greifswald, Greifswald, Deutschland

E-Mail: winfried.barthlen@uni-greifswald.de

D. von Schweinitz

Kinderchirurgische Klinik und Poliklinik, Dr. von Haunersches

Kinderspital, München, Deutschland

E-Mail: dietrich.schweinitz@med.uni-muenchen.de

\section{Therapie}

Bei Symptomen und zur histologischen Abklärung wird eine lokale Exzision der Zyste empfohlen, was in der Regel problemlos gelingt. Eine Pankreasresektion ist nur sehr selten notwendig (Wiersch und Gittes 2014).

\section{Duktäre Retentionszysten}

Diese entstehen durch eine Gangaufweitung bei Obstruktion oder chronischer Pankreatitis. Deshalb ist hier eine lokale Exzision nicht möglich. Präoperativ ist eine gute Darstellung der Verbindung zum Gangsystem mittels MRCP oder ERCP, ggf. mit Sphinkterotomie notwendig.

\section{Therapie}

Die operative Therapie besteht - neben einer Beseitigung der Obstruktion - in einer Roux-Y-Pankreato- oder Zystojejunostomie oder einer Resektion des betroffenen Pankreasabschnitts (Wiersch und Gittes 2014; Dzakovic und Superina 2012).

\section{Literatur}

Dzakovic A, Superina R (2012) Acute and chronic pancreatitis: surgical management. Semin Pediatr Surg 21(3):266-271

Wiersch J, Gittes GK (2014) Lesions of the pancreas. In: Holcomb GWIII, Murphy JP, Ostlie DJ (Hrsg) Ashraft's pediatric surgery. Elsevier Saunders, Philadelphia, S 636-647 